07.04 - Paediatric respiratory infection and immun.

\title{
11880
}

\section{Assessing polymicrobial interactions in a 3D primary airway epithelial cell model}

Bacteria, Viruses, Epithelial cell

\author{
M. W. P. Poh ${ }^{1}$, A. Fonceca ${ }^{2}$, A. Kicic ${ }^{3}$, M. Everard ${ }^{1}$
}

${ }^{1}$ University of Western Australia - Perth (Australia), ${ }^{2}$ Department of Health, Western Australia - Perth (Australia), ${ }^{3}$ Telethon Kids Institute - Perth (Australia)

\section{Introduction}

Mixed viral-bacterial aetiology is common in lower respiratory tract infections of children. However the pathogenic mechanisms involved are poorly understood. Using primary airway epithelial cells (AEC) derived from children which were then differentiated by growing at the Air-Liquid Interface (ALI), we infected/inoculated Respiratory Syncytial Virus (RSV) and nontypeable Haemophilus influenzae (NTHi) to assess the interaction between viral infection, NTHi biofilm and AEC. We hypothesised that an interaction occurs between RSV and NTHi manifested by enhanced formation of biofilms following viral infection, and release of planktonic NTHi from established biofilms following introduction of RSV.

\section{Methods}

Red fluorescent-tagged RSV (A2 strain, rrRSV-BN1, 1 $10^{6} \mathrm{pfu}$ ) and green fluorescent-tagged NTHi (86-028 $\mathrm{NP}, 2.5 \times 10^{7} \mathrm{CFU}$ ) were inoculated individually, concurrently or sequentially onto fully differentiated AEC grown on Transwell inserts (Corning). Cultures were left for 7 days. Insert surfaces were fixed, excised, mounted and overlayed with coverslips. Five confocal microscopy field-of-views at 200x magnification were obtained (Nikon C2+ system) and $1.2 \mu \mathrm{m}$ Z-stacks thickness used to reconstruct 3-dimensional images. Biomass thickness, surface area, and surface area to biomass ratio were determined using the COMSTAT program.

\section{Results}

No red fluorescence arising from RSV infection and replication were detected in epithelial cells precolonised by NTHi. However when infected earlier with RSV, NTHi were able to colonise and form biofilms on epithelial cells.

\section{Conclusions}


Prior NTHi colonisation of respiratory epithelium appears to shield against a secondary RSV infection, but not vice versa 